\title{
MATRIX-VARIATE KUMMER-BETA DISTRIBUTION
}

\author{
DAYA K. NAGAR and ARJUN K. GUPTA
}

(Received 17 January 2000; revised 23 May 2001)

Communicated by V. Stefanov

\begin{abstract}
This paper proposes matrix variate generalization of Kummer-Beta family of distributions which has been studied recently by $\mathrm{Ng}$ and $\mathrm{Kotz}$. This distribution is an extension of Beta distribution. Its characteristic function has been derived and it is shown that the distribution is orthogonally invariant. Some results on distribution of random quadratic forms have also been derived.
\end{abstract}

2000 Mathematics subject classification: primary 62H10; secondary 62E15.

Keywords and phrases: confluent hypergeometric function, invariant polynomial, Kummer-Beta distribution, matrix variate, transformation, zonal polynomial.

\section{Introduction}

Armero and Bayarri [1,2] derived Kummer distribution as a posterior distribution of certain basic parameters in a Bayesian analysis of $M / M / \infty$ queuing system. A random variable $X$ is said to have Kummer distribution if its probability density function (p.d.f.) is of the form

$$
f(x \mid \alpha, \beta, \gamma, \delta)=c(\alpha, \beta, \gamma, \delta) x^{\alpha-1}(1+\delta x)^{-\gamma} e^{-\beta x},
$$

where $x>0, \alpha>0, \beta>0, \delta>0$ and $-\infty<\gamma<\infty$. The normalizing constant is

$$
\begin{aligned}
\{c(\alpha, \beta, \gamma, \delta)\}^{-1} & =\int_{0}^{\infty} x^{\alpha-1}(1+\delta x)^{-\gamma} e^{-\beta x} d x \\
& =\delta^{-\alpha} \Gamma(\alpha) U(\alpha, \alpha+1-\gamma, \beta / \delta),
\end{aligned}
$$

where $U(a, b, z)$ is one of the two Kummer's functions (a confluent hypergeometric function). For $\gamma=0$, the above distribution reduces to a Gamma distribution with

(C) 2002 Australian Mathematical Society $1446-7887 / 2000 \$ A 2.00+0.00$ 
shape parameter $\alpha$ and scale parameter $\beta$. For $\alpha=\nu_{1} / 2, \beta=0, \gamma=\left(\nu_{1}+\nu_{2}\right) / 2$ and $\delta=1$, Kummer distribution reduces to $F$-distribution with $\left(\nu_{1}, \nu_{2}\right)$ degrees of freedom.

Recently, following Armero and Bayarri [1, 2], $\mathrm{Ng}$ and Kotz [11] proposed and studied two families of Kummer-Gamma and Kummer-Beta distributions. The random variable $U$ with the p.d.f.

$$
K(\alpha, \beta, \lambda) u^{\alpha-1}(1-u)^{\beta-1} e^{-\lambda u}, \quad 0<u<1,
$$

where $\alpha>0, \beta>0,-\infty<\lambda<\infty$ and

$$
\{K(\alpha, \beta, \lambda)\}^{-1}=\frac{\Gamma(\alpha) \Gamma(\beta)}{\Gamma(\alpha+\beta)}{ }_{1} F_{1}(\alpha, \alpha+\beta ;-\lambda),
$$

where ${ }_{1} F_{1}$ is the confluent hypergeometric function, is said to have Kummer-Beta distribution with parameters $(\alpha, \beta, \lambda)$. Independently, Gordy [7] has also defined Kummer-Beta distribution in relation to the problem of common value auction. This distribution is an extension of Beta distribution, and for $\alpha<1$ (and certain values of the parameter $\lambda$ ) yields bimodal distribution on finite range.

In this article we propose and study matrix variate generalization of (1.1). In Section 2, we give some well-known results and definitions. We define the matrix variate Kummer-Beta distribution in Section 3. Several of its properties including characteristic function, marginal distribution, moments are derived in Section 4.

\section{Preliminaries}

We begin with a brief review of some definitions and notations. We adhere to standard notations. See, for example, Constantine [4], Davis [5, 6] and Chikuse [3]. Throughout, $\kappa, \lambda, \phi$ and $\rho$ are partitions of the non-negative integers $k, l, f=k+l$ and $r$ respectively. The zonal polynomial of the symmetric $p \times p$ matrix $X$ corresponding to the partition $\kappa$ will be denoted by $C_{\kappa}(X)$. Davis $[5,6]$ has defined and studied a class of polynomials $C_{\phi}^{\kappa, \lambda}(X, Y)$ of $p \times p$ symmetric matrix arguments $X$ and $Y$, which are invariant under the transformation $(X, Y) \rightarrow\left(H X H^{\prime}, H Y H^{\prime}\right), H \in O(p)$. The following results pertaining to invariant polynomials will be used in subsequent sections:

$$
C_{\phi}^{\kappa, \lambda}(X, X)=\theta_{\phi}^{\kappa, \lambda} C_{\phi}(X)
$$

where $\theta_{\phi}^{\kappa, \lambda}=C_{\phi}^{\kappa, \lambda}\left(I_{p}, I_{p}\right) / C_{\phi}\left(I_{p}\right)$. Further,

$$
C_{\phi}^{\kappa, \lambda}\left(X, I_{p}\right)=\theta_{\phi}^{\kappa, \lambda} \frac{C_{\phi}\left(I_{p}\right) C_{\kappa}(X)}{C_{\kappa}\left(I_{p}\right)}, \quad C_{\kappa}^{\kappa, 0}(X, Y) \equiv C_{\kappa}(X),
$$


and

$$
C_{\kappa}(X) C_{\lambda}(Y)=\sum_{\phi \in \kappa \cdot \lambda} \theta_{\phi}^{\kappa, \lambda} C_{\phi}^{\kappa, \lambda}(X, Y)
$$

where $\phi \in \kappa \cdot \lambda$ denotes that irreducible representation of $G l(p, R)$, the group of $p \times p$ real invertible matrices, indexed by $2 \phi$, appears in the decomposition of the tensor product $2 \kappa \otimes 2 \lambda$ of the irreducible representations indexed by $2 \kappa$ and $2 \lambda$. The integrals involving invariant polynomials are

$$
\begin{aligned}
& \int_{0<R<I} \operatorname{det}(R)^{t-(p+1) / 2} \operatorname{det}(I-R)^{u-(p+1) / 2} C_{\phi}^{\kappa, \lambda}(R, I-R) d R \\
& =\frac{\Gamma_{p}(t, \kappa) \Gamma_{p}(u, \lambda)}{\Gamma_{p}(t+u, \phi)} \theta_{\phi}^{\kappa, \lambda} C_{\phi}(I), \\
& \int_{0<R<I} \operatorname{det}(R)^{t-(p+1) / 2} \operatorname{det}(I-R)^{u-(p+1) / 2} C_{\phi}^{\kappa, \lambda}(A R, B R) d R \\
& =\frac{\Gamma_{p}(t, \phi) \Gamma_{p}(u)}{\Gamma_{p}(t+u, \phi)} C_{\phi}^{\kappa, \lambda}(A, B),
\end{aligned}
$$

and

$$
\begin{gathered}
\int_{0<R<l} \operatorname{det}(R)^{t-(p+1) / 2} \operatorname{det}(I-R)^{u-(p+1) / 2} C_{\phi}^{\kappa, \lambda}\left(A R A^{\prime}, B\right) d R \\
=\frac{\Gamma_{p}(t, \kappa) \Gamma_{p}(u)}{\Gamma_{p}(t+u, \kappa)} C_{\phi}^{\kappa, \lambda}\left(A A^{\prime}, B\right) .
\end{gathered}
$$

In expressions (2.4), (2.5) and (2.6), $\Gamma_{p}(a, \rho)$ is defined by $\Gamma_{p}(a, \rho)=(a)_{\rho} \Gamma_{p}(a)$, with $(a)_{\rho}=\prod_{j=1}^{p}(a-(j-1) / 2)_{r_{j}}$,

$$
\Gamma_{p}(a, \rho)=\pi^{p(p-1) / 4} \prod_{j=1}^{p} \Gamma\left[a+r_{j}-(j-1) / 2\right], \quad \operatorname{Re}(a)>(p-1) / 2-r_{p},
$$

where $\rho=\left(r_{1}, \ldots, r_{p}\right), r_{1} \geq \cdots \geq r_{p} \geq 0, r_{1}+\cdots+r_{p}=r$. Note that $\Gamma_{p}(a, 0)=$ $\Gamma_{p}(a)$, which is multivariate gamma function given by

$$
\Gamma_{p}(a)=\pi^{p(p-1) / 4} \prod_{j=1}^{p} \Gamma[a-(j-1) / 2], \quad \operatorname{Re}(a)>(p-1) / 2 .
$$

The generalized hypergeometric functions of one matrix and two matrices are defined by

$$
{ }_{r} F_{s}\left(a_{1}, \ldots, a_{r} ; b_{1}, \ldots, b_{s} ; X\right)=\sum_{k=0}^{\infty} \sum_{\kappa} \frac{\left(a_{1}\right)_{\kappa} \cdots\left(a_{r}\right)_{\kappa}}{\left(b_{1}\right)_{\kappa} \cdots\left(b_{s}\right)_{\kappa}} \frac{C_{\kappa}(X)}{k !}
$$


and

$$
{ }_{r} F_{s}^{(p)}\left(a_{1}, \ldots, a_{r} ; b_{1}, \ldots, b_{s} ; X, Y\right)=\sum_{k=0}^{\infty} \sum_{\kappa} \frac{\left(a_{1}\right)_{\kappa} \cdots\left(a_{r}\right)_{\kappa}}{\left(b_{1}\right)_{\kappa} \cdots\left(b_{s}\right)_{\kappa}} \frac{C_{k}(X) C_{k}(Y)}{C_{k}\left(I_{p}\right) k !}
$$

respectively, where $a_{i}, i=1, \ldots, r ; b_{j}, j=1, \ldots, s$, are arbitrary complex numbers, $X(p \times p)$ and $Y(p \times p)$ are complex symmetric matrices and $\sum_{\kappa}$ denotes summation over all partitions $\kappa$. Conditions for convergence of these series are available in the literature. From (2.7) it follows that

$$
{ }_{0} F_{0}(X)=\sum_{k=0}^{\infty} \sum_{\kappa} \frac{C_{k}(X)}{k !}=\sum_{k=0}^{\infty} \frac{(\operatorname{tr} X)^{k}}{k !}=\operatorname{etr}(X)
$$

and

$$
{ }_{1} F_{1}(a ; b ; X)=\sum_{k=0}^{\infty} \sum_{\kappa} \frac{(a)_{\kappa}}{(b)_{k}} \frac{C_{k}(X)}{k !} .
$$

The integral representation of the confluent hypergeometric function ${ }_{1} F_{1}$ is given by

$$
\begin{aligned}
{ }_{1} F_{1}(a ; b ; X)= & \frac{\Gamma_{p}(b)}{\Gamma_{p}(a) \Gamma_{p}(b-a)} \int_{0<R<l_{p}} \operatorname{etr}(R X) \operatorname{det}(R)^{a-(p+1) / 2} \\
& \times \operatorname{det}\left(I_{p}-R\right)^{b-a-(p+1) / 2} d R
\end{aligned}
$$

where $\operatorname{Re}(a)>(p-1) / 2, \operatorname{Re}(b-a)>(p-1) / 2$. Next, we give definitions of matrix variate Gamma and Beta distributions (Gupta and Nagar [9]).

DEFINITION 2.1. A random symmetric positive definite matrix $W$ is said to follow a matrix variate Gamma distribution, denoted as $W \sim G_{p}(a, C)$, if its p.d.f. is

$$
\left\{\Gamma_{p}(a) \operatorname{det}(C)^{-a}\right\}^{-1} \operatorname{etr}(-C W) \operatorname{det}(W)^{a-(p+1) / 2}, \quad W>0,
$$

where $a>(p-1) / 2$ and $C$ is a $p \times p$ symmetric positive definite non-random matrix.

DEFINITION 2.2. A $p \times p$ random symmetric positive definite matrix $X$ is said to have a matrix variate Beta type I distribution with parameters $(a, b)$, denoted as $X \sim B_{p}^{I}(a, b)$, if its p.d.f. is given by

$$
\left\{\beta_{p}(a, b)\right\}^{-1} \operatorname{det}(X)^{a-(p+1) / 2} \operatorname{det}\left(I_{p}-X\right)^{b-(p+1) / 2}, \quad 0<X<I_{p},
$$

where $a>(p-1) / 2, b>(p-1) / 2$, and $\beta_{p}(a, b)$ is the multivariate beta function given by

$$
\beta_{p}(a, b)=\frac{\Gamma_{p}(a) \Gamma_{p}(b)}{\Gamma_{p}(a+b)}
$$


DEFINITION 2.3. A $p \times p$ random symmetric positive definite matrix $Y$ is said to have a matrix variate Beta type II distribution with parameters $(a, b)$, denoted as $Y \sim B_{p}^{l l}(a, b)$, if its p.d.f. is given by

$$
\left\{\beta_{p}(a, b)\right\}^{-1} \operatorname{det}(Y)^{a-(p+1) / 2} \operatorname{det}\left(I_{p}+Y\right)^{-(a+b)}, \quad Y>0,
$$

where $a>(p-1) / 2, b>(p-1) / 2$, and $\beta_{p}(a, b)$ is the multivariate beta function.

\section{Matrix variate Kummer-Beta distribution}

The corresponding matrix variate generalization of $(1.1)$ is defined as follows:

DEFINITION 3.1. A $p \times p$ random symmetric positive definite matrix $U$ is said to have a matrix variate Kummer-Beta distribution with parameters $\alpha, \beta$ and $\Lambda$, denoted as $U \sim K B_{p}(\alpha, \beta, \Lambda)$, if its p.d.f. is given by

$$
K(\alpha, \beta, \Lambda) \operatorname{etr}(-\Lambda U) \operatorname{det}(U)^{\alpha-(p+1) / 2} \operatorname{det}\left(I_{p}-U\right)^{\beta-(p+1) / 2}, \quad 0<U<I_{p},
$$

where $\alpha>(p-1) / 2, \beta>(p-1) / 2, \Lambda(p \times p)$ is symmetric and $K(\alpha, \beta, \Lambda)$ is the normalizing constant.

The normalizing constant in (3.1) is given as

$$
\begin{aligned}
\{K(\alpha, \beta, \Lambda)\}^{-1} & =\int_{0<U<l_{p}} \operatorname{etr}(-\Lambda U) \operatorname{det}(U)^{\alpha-(p+1) / 2} \operatorname{det}\left(I_{p}-U\right)^{\beta-(p+1) / 2} d U \\
& =\frac{\Gamma_{p}(\alpha) \Gamma_{p}(\beta)}{\Gamma_{p}(\alpha+\beta)}{ }_{1} F_{1}(\alpha ; \alpha+\beta ;-\Lambda)
\end{aligned}
$$

where ${ }_{1} F_{1}$ is the confluent hypergeometric function of matrix argument.

It may be noted here that for $\Lambda=0$, Kummer-Beta distribution reduces to a Beta type I distribution.

By means of a bilinear transformation of the random matrix $U$, a generalized matrix variate Kummer-Beta distribution is generated as given in the following theorem.

THEOREM 3.1. Let $U \sim K B_{p}(\alpha, \beta, \Lambda)$. Then for given $p \times p$ symmetric matrices $\Psi>0$ and $\Omega(>\Psi)$, the random matrix $X(p \times p)$ defined by

$$
X=(\Omega-\Psi)^{1 / 2} U(\Omega-\Psi)^{1 / 2}+\Psi
$$

has the p.d.f.

$$
C(\alpha, \beta, \Theta, \Omega, \Psi) \operatorname{etr}(-\Theta X) \operatorname{det}(X-\Psi)^{\alpha-(p+1) / 2} \operatorname{det}(\Omega-X)^{\beta-(p+1) / 2},
$$


where $\Psi<X<\Omega, \Theta=(\Omega-\Psi)^{-1 / 2} \Lambda(\Omega-\Psi)^{-1 / 2}$ and

$$
\begin{aligned}
C(\alpha, \beta, \Theta, \Omega, \Psi)= & K\left(\alpha, \beta,(\Omega-\Psi)^{1 / 2} \Theta(\Omega-\Psi)^{1 / 2}\right) \operatorname{etr}(\Theta \Psi) \\
& \times \operatorname{det}(\Omega-\Psi)^{-(\alpha+\beta)+(p+1) / 2} .
\end{aligned}
$$

PROOF. The Jacobian of the transformation (3.3) is $J(U \rightarrow X)=\operatorname{det}(\Omega-$ $\Psi)^{-(p+1) / 2}$. Hence, the p.d.f. of $U$ is transformed to the p.d.f. of $X$ given by (3.4).

DEFINITION 3.2. A $p \times p$ random symmetric positive definite matrix $X$ is said to have a generalized matrix variate Kummer-Beta distribution with parameters $\alpha, \beta, \Theta$; $\Omega, \Psi$ denoted by $X \sim G K B_{p}(\alpha, \beta, \Theta, \Omega, \Psi)$, if its p.d.f. is given by

$$
C(\alpha, \beta, \Theta, \Omega, \Psi) \operatorname{etr}(-\Theta X) \operatorname{det}(X-\Psi)^{\alpha-(p+1) / 2} \operatorname{det}(\Omega-X)^{\beta-(p+1) / 2},
$$

$\Psi<X<\Omega$, where

$$
\begin{aligned}
C(\alpha, \beta, \Theta, \Omega, \Psi)= & K\left(\alpha, \beta,(\Omega-\Psi)^{1 / 2} \Theta(\Omega-\Psi)^{1 / 2}\right) \operatorname{etr}(\Theta \Psi) \\
& \times \operatorname{det}(\Omega-\Psi)^{-(\alpha+\beta)+(p+1) / 2}
\end{aligned}
$$

When $\Psi=0$ and $\Omega=I_{p}$, the above definition yields the standard Kummer-Beta distribution. Further if $X \sim G K B_{p}(\alpha, \beta, \Theta, \Omega, \Psi)$, then $(\Omega-\Psi)^{-1 / 2}(X-\Psi)(\Omega-$ $\Psi)^{-1 / 2} \sim K B_{p}\left(\alpha, \beta,(\Omega-\Psi)^{1 / 2} \Theta(\Omega-\Psi)^{1 / 2}\right)$.

\section{Properties}

In this section we study some properties of the random matrix distributed as matrix variate Kummer-Beta.

THEOREM 4.1. Let $U \sim K B_{p}(\alpha, \beta, \Lambda)$ and $A(p \times p)$ be a constant nonsingular matrix. Then $A U A^{\prime} \sim G K B_{p}\left(\alpha, \beta,\left(A^{-1}\right)^{\prime} \Lambda A^{-1}, A A^{\prime}, 0\right)$.

PROOF. In the p.d.f. (3.1) of $U$, making the transformation $X=A U A^{\prime}$ with Jacobian $J(U \rightarrow X)=\operatorname{det}\left(A A^{\prime}\right)^{-(p+1) / 2}$, the density of $X$ is obtained as

$$
\begin{aligned}
& K(\alpha, \beta, \Lambda) \operatorname{det}\left(A A^{\prime}\right)^{-\alpha-\beta+(p+1) / 2} \operatorname{etr}\left(-\left(A^{-1}\right)^{\prime} \Lambda A^{-1} X\right) \\
& \quad \times \operatorname{det}(X)^{\alpha-(p+1) / 2} \operatorname{det}\left(A A^{\prime}-X\right)^{\beta-(p+1) / 2}, \quad 0<X<A A^{\prime},
\end{aligned}
$$

which is the desired result.

In the next theorem, it is shown that the matrix variate Kummer-Beta distribution is orthogonally invariant. 
THEOREM 4.2. Let $U \sim K B_{p}\left(\alpha, \beta, \lambda I_{p}\right)$, and $H(p \times p)$ be an orthogonal matrix whose elements are either constants or random variables distributed independent of $U$. Then, the distribution of $U$ is invariant under the transformation $U \rightarrow H U H^{\prime}$, and is independent of $H$ in the latter case.

PrOOF. First, let $H$ be a constant orthogonal matrix. Then, from Theorem 4.1, $H U H^{\prime} \sim K B_{p}\left(\alpha, \beta, \lambda I_{p}\right)$ since $H H^{\prime}=I_{p}$. If, however, $H$ is a random orthogonal matrix, then the conditional distribution of $H U H^{\prime} \mid H \sim K B_{p}\left(\alpha, \beta, \lambda I_{p}\right)$. Since this distribution does not depend on $H, H U H^{\prime} \sim K B_{p}\left(\alpha, \beta, \lambda I_{p}\right)$.

The characteristic functions of $U$ is now obtained in the following theorem.

THEOREM 4.3. Let $U \sim K B_{p}(\alpha, \beta, \Lambda)$. Then the characteristic function of $U=$ $\left(u_{i j}\right)$, that is, the joint characteristic function of $u_{11}, u_{12}, \ldots, u_{p p}$ is

$$
\phi_{U}(Z)=\frac{{ }_{1} F_{1}(\alpha ; \alpha+\beta ; \iota Z-\Lambda)}{{ }_{1} F_{1}(\alpha ; \alpha+\beta ;-\Lambda)},
$$

where $Z=Z^{\prime}(p \times p)=\left(\left(1+\delta_{i j}\right) z_{i j} / 2\right)$ and $\iota=\sqrt{-1}$.

ProOF. By definition,

$$
\begin{aligned}
\phi_{U}(Z) & =E[\operatorname{etr}(\iota Z U)] \\
& =K(\alpha, \beta, \Lambda) \int_{0<U<l_{p}} \operatorname{etr}(\iota Z U-\Lambda U) \operatorname{det}(U)^{\alpha-(p+1) / 2} \operatorname{det}\left(I_{p}-U\right)^{\beta-(p+1) / 2} d U \\
& =\frac{K(\alpha, \beta, \Lambda)}{K(\alpha, \beta, \Lambda-\iota Z)}=\frac{{ }_{1} F_{1}(\alpha ; \alpha+\beta ; \iota Z-\Lambda)}{{ }_{1} F_{1}(\alpha ; \alpha+\beta ;-\Lambda)} .
\end{aligned}
$$

The last equality follows from (3.2).

It may be noted here that if $X \sim G K B_{p}(\alpha, \beta, \Theta, \Omega, \Psi)$, then the characteristic function of $X$ can be obtained from Theorem 4.3. Since $X=(\Omega-\Psi)^{1 / 2} U(\Omega-$ $\Psi)^{1 / 2}+\Psi$, where $U \sim K B_{p}\left(\alpha, \beta,(\Omega-\Psi)^{1 / 2} \Theta(\Omega-\Psi)^{1 / 2}\right)$, we have

$$
\begin{aligned}
\phi_{X}(Z) & =E[\operatorname{etr}(\iota Z X)]=E\left[\operatorname{etr}\left\{\iota Z\left((\Omega-\Psi)^{1 / 2} U(\Omega-\Psi)^{1 / 2}+\Psi\right)\right\}\right] \\
& =\operatorname{etr}(\iota Z \Psi) E\left[\operatorname{etr}\left\{\iota(\Omega-\Psi)^{1 / 2} Z(\Omega-\Psi)^{1 / 2} U\right\}\right] \\
& =\operatorname{etr}(\iota Z \Psi) \phi_{U}\left((\Omega-\Psi)^{1 / 2} Z(\Omega-\Psi)^{1 / 2}\right) \\
& =\operatorname{etr}(\iota Z \Psi) \frac{{ }_{1} F_{1}\left(\alpha ; \alpha+\beta ; \iota(\Omega-\Psi)^{1 / 2}(\iota Z-\Theta)(\Omega-\Psi)^{1 / 2}\right)}{{ }_{1} F_{1}\left(\alpha ; \alpha+\beta ;-(\Omega-\Psi)^{1 / 2} \Theta(\Omega-\Psi)^{1 / 2}\right)} .
\end{aligned}
$$

THEOREM 4.4. If $U \sim K B_{p}(\alpha, \beta, \Lambda)$, then $I_{p}-U \sim K B_{p}(\beta, \alpha,-\Lambda)$. 
Let $U=\left(\begin{array}{ll}U_{11} & U_{12} \\ U_{21} & U_{22}\end{array}\right), U_{11}(q \times q)$ and $U_{22.1}=U_{22}-U_{21} U_{11}^{-1} U_{12}$. If $U \sim K B_{p}(\alpha, \beta, 0)$, then $U_{11}$ and $U_{22.1}$ are independent, $U_{11} \sim B_{q}^{I}(\alpha, \beta)$ and $U_{22.1} \sim B_{p-q}^{I}(\alpha-q / 2, \beta)$. Further, if $\Lambda=\left(\begin{array}{cc}\Lambda_{11} & 0 \\ 0 & \Lambda_{22}\end{array}\right), \Lambda_{11}(q \times q), \Lambda_{22}=0$, then also $U_{11}$ and $U_{22.1}$ are independent, $U_{11} \sim K B_{q}\left(\alpha, \beta, \Lambda_{11}\right)$ and $U_{22 \cdot 1} \sim B_{p-q}^{I}(\alpha-q / 2, \beta)$. But if $\Lambda_{22} \neq 0$, then $U_{11}$ and $U_{22.1}$ are not independent. For this consider $U \sim K B_{p}\left(\alpha, \beta, I_{p}\right)$. From the partition of $U$, we have

$$
\begin{gathered}
\operatorname{det}(U)=\operatorname{det}\left(U_{11}\right) \operatorname{det}\left(U_{22 \cdot 1}\right) \\
\operatorname{det}\left(I_{p}-U\right)=\operatorname{det}\left(I_{q}-U_{11}\right) \operatorname{det}\left(I_{p-q}-U_{22 \cdot 1}-U_{21} U_{11}^{-1}\left(I_{q}-U_{11}\right)^{-1} U_{12}\right)
\end{gathered}
$$

and

$$
\operatorname{tr}(U)=\operatorname{tr}\left(U_{11}\right)+\operatorname{tr}\left(U_{22.1}\right)+\operatorname{tr}\left(U_{21} U_{11}^{-1} U_{12}\right) .
$$

Now making the transformation

$$
U_{11}=U_{11}, \quad X=U_{21} U_{11}^{-1 / 2} \quad \text { and } \quad U_{22 \cdot 1}=U_{22}-U_{21} U_{11}^{-1} U_{12}=U_{22}-X X^{\prime}
$$

with Jacobian $J\left(U_{11}, U_{22}, U_{21} \rightarrow U_{11}, U_{22.1}, X\right)=\operatorname{det}\left(U_{11}\right)^{(p-q) / 2}$ and substituting (4.1)-(4.3) in the density of $U$, we get the joint density of $U_{11}, U_{22.1}$, and $X$ as

$$
\begin{aligned}
K(\alpha, & \left.\beta, I_{p}\right) \operatorname{det}\left(U_{11}\right)^{\alpha-(q+1) / 2} \operatorname{det}\left(I_{q}-U_{11}\right)^{\beta-(p+1) / 2} \\
& \times \operatorname{det}\left(I_{p-q}-\left(I_{p-q}-U_{22.1}\right)^{-1} X\left(I_{q}-U_{11}\right)^{-1} X^{\prime}\right)^{\beta-(p+1) / 2} \operatorname{det}\left(U_{22 \cdot 1}\right)^{\alpha-(p+1) / 2} \\
& \times \operatorname{det}\left(I_{p-q}-U_{22 \cdot 1}\right)^{\beta-(p+1) / 2} \exp \left\{-\operatorname{tr}\left(U_{11}\right)-\operatorname{tr}\left(U_{22 \cdot 1}\right)-\operatorname{tr}\left(X X^{\prime}\right)\right\} .
\end{aligned}
$$

Now making the transformation $Y=\left(I_{p-q}-U_{22 \cdot 1}\right)^{-1 / 2} X\left(I_{q}-U_{11}\right)^{-1 / 2}$ with Jacobian $J(X \rightarrow Y)=\operatorname{det}\left(I_{p-q}-U_{22.1}\right)^{q / 2} \operatorname{det}\left(I_{q}-U_{11}\right)^{(p-q) / 2}$ and integrating $Y$ we get the joint density of $U_{11}$ and $U_{22.1}$ as

$$
\begin{aligned}
K(\alpha, & \left.\beta, I_{p}\right) \operatorname{det}\left(U_{11}\right)^{\alpha-(q+1) / 2} \operatorname{det}\left(I_{q}-U_{11}\right)^{\beta-(q+1) / 2} \operatorname{det}\left(U_{22 \cdot 1}\right)^{\alpha-(p+1) / 2} \\
& \times \operatorname{det}\left(I_{p-q}-U_{22 \cdot 1}\right)^{\beta-(p-q+1) / 2} e^{\left(-\operatorname{tr}\left(U_{11}\right)-\operatorname{tr}\left(U_{22.1}\right)\right)} g(A, B),
\end{aligned}
$$

where

$$
\begin{aligned}
g(A, B) & =\int_{I_{p-q}-Y Y^{\prime}>0} e^{\left(-\operatorname{tr}\left(A Y B Y^{\prime}\right)\right\}} \operatorname{det}\left(I_{p-q}-Y Y^{\prime}\right)^{\beta-(p+1) / 2} d Y \\
& =\left\{\begin{array}{lll}
\int_{0<Z<I_{p-q}} \int_{Y Y^{\prime}=Z} \operatorname{det}\left(I_{p-q}-Y Y^{\prime}\right)^{\beta-(p+1) / 2}{ }_{0} F_{0}\left(-Y^{\prime} A Y B\right) d Y, & p-q \leq q ; \\
\int_{0<Z<I_{q}} \int_{Y^{\prime} Y=Z}^{\operatorname{det}\left(I_{q}-Y^{\prime} Y\right)^{\beta-(p+1) / 2}{ }_{0} F_{0}\left(-A Y B Y^{\prime}\right) d Y,} & p-q>q,
\end{array}\right.
\end{aligned}
$$

with $A=I_{p-q}-U_{22.1}$ and $B=I_{q}-U_{11}$. Since $g(A, B)=g\left(A, H B H^{\prime}\right), H \in O(q)$, integrating $H$ in $g\left(A, H B H^{\prime}\right)$ we obtain, for $p-q \leq q$,

$$
g(A, B)=\int_{0<Z<I_{p-q}} \int_{Y Y^{\prime}=Z} \operatorname{det}\left(I_{p-q}-Y Y^{\prime}\right)^{\beta-(p+1) / 2}{ }_{0} F_{0}^{(q)}\left(-Y^{\prime} A Y, B\right) d Y
$$




$$
\begin{aligned}
= & \frac{\pi^{q(p-q) / 2}}{\Gamma_{p-q}(q / 2)} \int_{0<Z<I_{p-q}} \operatorname{det}(Z)^{(q-p+q-1) / 2} \operatorname{det}\left(I_{p-q}-Z\right)^{\beta-(p+1) / 2} \\
& \times{ }_{0} F_{0}^{(q)}(-A Z, B) d Z \\
= & \frac{\pi^{q(p-q) / 2} \Gamma_{p-q}(\beta-q / 2)}{\Gamma_{p-q}(\beta)} F_{1}^{(q)}\left(q / 2 ; \beta ;-\left(I_{p-q}-U_{22 \cdot 1}\right), I_{q}-U_{11}\right) .
\end{aligned}
$$

Substituting $g(A, B)$ in (4.4) we get the joint density of $U_{11}$ and $U_{22.1}$ as

$$
\begin{aligned}
K(\alpha, \beta, & \left.I_{p}\right) e^{\left\{-\operatorname{tr}\left(U_{11}\right)\right\}} \operatorname{det}\left(U_{11}\right)^{\alpha-(q+1) / 2} \operatorname{det}\left(I_{q}-U_{11}\right)^{\beta-(q+1) / 2} \\
& \times e^{\left[-\operatorname{tr}\left(U_{22 \cdot 1}\right)\right\}} \operatorname{det}\left(U_{22 \cdot 1}\right)^{\alpha-(p+1) / 2} \operatorname{det}\left(I_{p-q}-U_{22 \cdot 1}\right)^{\beta-(p-q+1) / 2} \\
& \times \frac{\pi^{q(p-q) / 2} \Gamma_{p-q}(\beta-q / 2)}{\Gamma_{p-q}(\beta)}{ }_{1} F_{1}^{(q)}\left(q / 2 ; \beta ;-\left(I_{p-q}-U_{22 \cdot 1}\right), I_{q}-U_{11}\right) .
\end{aligned}
$$

Clearly $U_{11}$ and $U_{22.1}$ are not independent. The marginal densities of $U_{11}$ and $U_{22.1}$ can be obtained by using results on invariant polynomials and hypergeometric functions. For $q \geq p-q$, expanding

$$
\begin{aligned}
{ }_{1} F_{1}^{(q)}\left(q / 2 ; \beta ;-A, I_{q}-U_{11}\right) & =\sum_{l=0}^{\infty} \sum_{\lambda} \frac{(q / 2)_{\lambda}}{(\beta)_{\lambda} l !}(-1)^{l} \frac{C_{\lambda}(A) C_{\lambda}\left(I_{q}-U_{11}\right)}{C_{\lambda}\left(I_{q}\right)}, \\
e^{\left[-\operatorname{tr}\left(U_{11}\right)\right]} & =\sum_{k=0}^{\infty} \sum_{\kappa} \frac{(-1)^{k}}{k !} C_{\kappa}\left(U_{11}\right)
\end{aligned}
$$

and using $C_{\kappa}\left(U_{11}\right) C_{\lambda}\left(I_{q}-U_{11}\right)=\sum_{\phi \in \kappa \cdot \lambda} \theta_{\phi}^{\kappa, \lambda} C_{\phi}^{\kappa, \lambda}\left(U_{11}, I_{q}-U_{11}\right)$ we have

$$
\begin{aligned}
& e^{\left[-\operatorname{tr}\left(U_{11}\right)\right)}{ }_{1} F_{1}^{(q)}\left(q / 2 ; \beta ;-A, I_{q}-U_{11}\right) \\
& =\sum_{k=0}^{\infty} \sum_{l=0}^{\infty} \sum_{\kappa} \sum_{\lambda} \frac{1}{k !} \frac{(q / 2)_{\lambda}}{(\beta)_{\lambda} l !}(-1)^{k+l} \frac{C_{\lambda}(A)}{C_{\lambda}\left(I_{q}\right)} \sum_{\phi \in \kappa-\lambda} \theta_{\phi}^{\kappa, \lambda} C_{\phi}^{\kappa, \lambda}\left(U_{11}, I_{q}-U_{11}\right) .
\end{aligned}
$$

Substituting (4.6) in (4.5) and integrating $U_{11}$ using (2.4) we obtain

$$
\begin{aligned}
\int_{0<U_{11}<I_{q}} \operatorname{det}\left(U_{11}\right)^{\alpha-(q+1) / 2} \operatorname{det}\left(I_{q}-U_{11}\right)^{\beta-(q+1) / 2} e^{\left.\mid-\operatorname{tr}\left(U_{11}\right)\right)} \\
\quad \times{ }_{1} F_{1}^{(q)}\left(q / 2 ; \beta ;-A, I_{q}-U_{11}\right) d U_{11} \\
=\sum_{k=0}^{\infty} \sum_{l=0}^{\infty} \sum_{\kappa} \sum_{\lambda} \frac{1}{k !} \frac{(q / 2)_{\lambda}}{(\beta)_{\lambda} l !}(-1)^{k+l} \frac{C_{\lambda}(A)}{C_{\lambda}\left(I_{q}\right)} \sum_{\phi \in \kappa \cdot \lambda} \theta_{\phi}^{\kappa, \lambda} \\
\quad \times \int_{0<U_{11}<I_{q}} \operatorname{det}\left(U_{11}\right)^{\alpha-(q+1) / 2} \operatorname{det}\left(I_{q}-U_{11}\right)^{\beta-(q+1) / 2} C_{\phi}^{\kappa, \lambda}\left(U_{11}, I_{q}-U_{11}\right) d U_{11} \\
=\sum_{k=0}^{\infty} \sum_{l=0}^{\infty} \sum_{\kappa} \sum_{\lambda} \frac{1}{k !} \frac{(q / 2)_{\lambda}}{(\beta)_{\lambda} l !}(-1)^{k+l} \frac{C_{\lambda}(A)}{C_{\lambda}\left(I_{q}\right)} \sum_{\phi \in \kappa \cdot \lambda}\left(\theta_{\phi}^{\kappa, \lambda}\right)^{2} \frac{\Gamma_{q}(\alpha, \kappa) \Gamma_{q}(\beta, \lambda)}{\Gamma_{q}(\alpha+\beta, \phi)} C_{\phi}\left(I_{q}\right) .
\end{aligned}
$$


Finally, the marginal density of $U_{22 \cdot 1}$ is derived as

$$
\begin{aligned}
& K\left(\alpha, \beta, I_{p}\right) \frac{\pi^{q(p-q) / 2} \Gamma_{p-q}(\beta-q / 2)}{\Gamma_{p-q}(\beta)} e^{i-\operatorname{tr}\left(U_{22-1}\right) l} \operatorname{det}\left(U_{22 \cdot 1}\right)^{\alpha-(p+1) / 2} \\
& \quad \times \operatorname{det}\left(I_{p-q}-U_{22 \cdot 1}\right)^{\beta-(p-q+1) / 2} \sum_{k=0}^{\infty} \sum_{l=0}^{\infty} \sum_{\kappa} \sum_{\lambda} \frac{1}{k !} \frac{(q / 2)_{\lambda}}{(\beta)_{\lambda} l !}(-1)^{k+l} \frac{C_{\lambda}(A)}{C_{\lambda}\left(I_{q}\right)} \\
& \quad \times \sum_{\phi \in \kappa \cdot \lambda}\left(\theta_{\phi}^{\kappa, \lambda}\right)^{2} \frac{\Gamma_{q}(\alpha, \kappa) \Gamma_{q}(\beta, \lambda)}{\Gamma_{q}(\alpha+\beta, \phi)} C_{\phi}\left(I_{q}\right) .
\end{aligned}
$$

Next, we derive moments of some functions of the random matrix $U$ distributed as matrix variate Kummer-Beta.

THEOREM 4.5. Let $U \sim K B_{p}(\alpha, \beta, \Lambda)$, then

(i) $E\left[\operatorname{det}(U)^{h}\right]=\frac{\Gamma_{p}(\alpha+h) \Gamma_{p}(\alpha+\beta)}{\Gamma_{p}(\alpha) \Gamma_{p}(\alpha+\beta+h)} \cdot \frac{{ }_{1} F_{1}(\alpha+h ; \alpha+\beta+h,-\Lambda)}{{ }_{1} F_{1}(\alpha ; \alpha+\beta,-\Lambda)}$,

$$
\operatorname{Re}(h)>-\alpha+(p-1) / 2 \text {; }
$$

(ii) $E\left[\operatorname{det}\left(I_{p}-U\right)^{h}\right]=\frac{\Gamma_{p}(\beta+h) \Gamma_{p}(\alpha+\beta)}{\Gamma_{p}(\beta) \Gamma_{p}(\alpha+\beta+h)} \cdot \frac{{ }_{1} F_{1}(\alpha ; \alpha+\beta+h,-\Lambda)}{{ }_{1} F_{1}(\alpha ; \alpha+\beta,-\Lambda)}$,

$$
\operatorname{Re}(h)>-\beta+(p-1) / 2,
$$

where ${ }_{1} F_{1}$ is the confluent hypergeometric function of matrix argument.

ProOF. (i) From the density (3.1) we have, for $\operatorname{Re}(h)>-\alpha+(p-1) / 2$,

$$
\begin{aligned}
E\left[\operatorname{det}(U)^{h}\right] & =K(\alpha, \beta, \Lambda) \int_{0<U<l_{p}} \operatorname{etr}(-\Lambda U) \operatorname{det}(U)^{\alpha+h-(p+1) / 2} \operatorname{det}\left(I_{p}-U\right)^{\beta-(p+1) / 2} d U \\
& =\frac{K(\alpha, \beta, \Lambda)}{K(\alpha+h, \beta, \Lambda)} \\
& =\frac{\Gamma_{p}(\alpha+h) \Gamma_{p}(\alpha+\beta)}{\Gamma_{p}(\alpha) \Gamma_{p}(\alpha+\beta+h)} \cdot \frac{{ }_{1} F_{1}(\alpha+h ; \alpha+\beta+h,-\Lambda)}{{ }_{1} F_{1}(\alpha ; \alpha+\beta,-\Lambda)} .
\end{aligned}
$$

(ii) Similar to the proof of (i).

From the density of $U$,

$$
\begin{aligned}
E\left[C_{\kappa}(A U)\right]=K & (\alpha, \beta, \Lambda) \int_{0<U<I_{p}} C_{\kappa}(A U) \operatorname{etr}(-\Lambda U) \\
& \times \cdot \operatorname{det}(U)^{\alpha-(p+1) / 2} \operatorname{det}\left(I_{p}-U\right)^{\beta-(p+1) / 2} d U .
\end{aligned}
$$

Expanding $\operatorname{etr}(-\Lambda U)$ in zonal polynomials and using results on invariant polynomials, we get

$$
C_{\kappa}(A U) \operatorname{etr}(-\Lambda U)=\sum_{l=0}^{\infty} \sum_{\lambda} \frac{(-1)^{l}}{l !} C_{\kappa}(A U) C_{\lambda}(\Lambda U)
$$




$$
=\sum_{l=0}^{\infty} \sum_{\lambda} \frac{(-1)^{l}}{l !} \sum_{\phi \in \kappa \cdot \lambda} \theta_{\phi}^{\kappa, \lambda} C_{\phi}^{\kappa, \lambda}(A U, \Lambda U) .
$$

Now substituting (4.8) in (4.7) and integrating $U$ using (2.5), we obtain

$$
\begin{aligned}
E\left[C_{\kappa}(A U)\right]= & K(\alpha, \beta, \Lambda) \sum_{l=0}^{\infty} \sum_{\lambda} \frac{(-1)^{l}}{l !} \sum_{\phi \in \kappa \cdot \lambda} \theta_{\phi}^{\kappa, \lambda} \int_{0<U<I_{p}} \operatorname{det}(U)^{\alpha-(p+1) / 2} \\
& \times \operatorname{det}\left(I_{p}-U\right)^{\beta-(p+1) / 2} C_{\phi}^{\kappa, \lambda}(A U, \Lambda U) d U \\
= & K(\alpha, \beta, \Lambda) \sum_{l=0}^{\infty} \sum_{\lambda} \frac{(-1)^{l}}{l !} \sum_{\phi \in \kappa \cdot \lambda} \theta_{\phi}^{\kappa, \lambda} \frac{\Gamma_{p}(\alpha, \phi) \Gamma_{p}(\beta)}{\Gamma_{p}(\alpha+\beta, \phi)} C_{\phi}^{\kappa, \lambda}(A, \Lambda) .
\end{aligned}
$$

Similarly,

$$
\begin{aligned}
E\left[C_{\kappa}\left(I_{p}-U\right)\right] & \\
= & K(\alpha, \beta, \Lambda) \operatorname{etr}(-\Lambda) \sum_{l=0}^{\infty} \sum_{\lambda} \frac{1}{l !} \sum_{\phi \in \kappa \cdot \lambda} \theta_{\phi}^{\kappa, \lambda} \int_{0<X<l_{p}} \operatorname{det}\left(I_{p}-X\right)^{\alpha-(p+1) / 2} \\
& \times \operatorname{det}(X)^{\beta-(p+1) / 2} C_{\phi}^{\kappa, \lambda}(X, \Lambda X) d X \\
= & K(\alpha, \beta, \Lambda) \operatorname{etr}(-\Lambda) \sum_{l=0}^{\infty} \sum_{\lambda} \frac{1}{l !} \sum_{\phi \in \kappa \cdot \lambda} \theta_{\phi}^{\kappa, \lambda} \frac{\Gamma_{p}(\beta, \phi) \Gamma_{p}(\alpha)}{\Gamma_{p}(\alpha+\beta, \phi)} C_{\phi}^{\kappa, \lambda}\left(I_{p}, \Lambda\right) \\
= & K(\alpha, \beta, \Lambda) \operatorname{etr}(-\Lambda) \sum_{l=0}^{\infty} \sum_{\lambda} \frac{1}{l !} \sum_{\phi \in \kappa \cdot \lambda}\left(\theta_{\phi}^{\kappa, \lambda}\right)^{2} \frac{\Gamma_{p}(\beta, \phi) \Gamma_{p}(\alpha)}{\Gamma_{p}(\alpha+\beta, \phi)} \frac{C_{\phi}\left(I_{p}\right)}{C_{\lambda}\left(I_{p}\right)} C_{\lambda}(\Lambda),
\end{aligned}
$$

where the last two steps have been obtained by using (2.5) and (2.2) respectively.

In the rest of the section we will derive density functions of certain random quadratic forms.

THEOREM 4.6. Let $X \sim B_{p}^{I}(a, b)$ and $U \sim K B_{p}(\alpha, \beta, \Lambda)$ be independent. Then the density of $Z=U^{1 / 2} X\left(U^{1 / 2}\right)^{\prime}$ is given by, for $0<Z<I_{p}$,

$$
\begin{aligned}
& \frac{\Gamma_{p}(a+b) \Gamma_{p}(\alpha+\beta)}{\Gamma_{p}(a) \Gamma_{p}(\alpha) \Gamma_{p}(b+\beta)}\left\{{ }_{1} F_{1}(\beta ; \alpha+\beta ; \Lambda)\right\}^{-1} \operatorname{det}(Z)^{a-(p+1) / 2} \operatorname{det}\left(I_{p}-Z\right)^{b+\beta-(p+1) / 2} \\
& \quad \times \sum_{k=0}^{\infty} \sum_{l=0}^{\infty} \sum_{\kappa} \sum_{\lambda} \frac{1}{k !} \frac{(a+b-\alpha)_{\lambda}}{l !} \sum_{\phi \in \kappa \cdot \lambda} \theta_{\phi}^{\kappa, \lambda} \frac{(\beta)_{\kappa}}{(b+\beta)_{\kappa}} C_{\phi}^{\kappa, \lambda}\left(\Lambda\left(I_{p}-Z\right), I_{p}-Z\right) .
\end{aligned}
$$

ProOF. The joint density of $X$ and $U$ is

$$
\begin{aligned}
& \left\{\beta_{p}(a, b)\right\}^{-1} K(\alpha, \beta, \Lambda) \operatorname{det}(X)^{a-(p+1) / 2} \operatorname{det}\left(I_{p}-X\right)^{b-(p+1) / 2} \operatorname{etr}(-\Lambda U) \\
& \quad \times \operatorname{det}(U)^{\alpha-(p+1) / 2} \operatorname{det}\left(I_{p}-U\right)^{\beta-(p+1) / 2}, \quad 0<U<I_{p}, 0<Y<I_{p}
\end{aligned}
$$


Making the transformation $Z=U^{1 / 2} X\left(U^{1 / 2}\right)^{\prime}$ with the Jacobian $J(X, U \rightarrow Z, U)=$ $\operatorname{det}(U)^{-(p+1) / 2}$ in (4.9) we get the joint density of $Z$ and $U$ as

$$
\begin{aligned}
& \frac{\Gamma_{p}(a+b) \Gamma_{p}(\alpha+\beta)}{\Gamma_{p}(a) \Gamma_{p}(b) \Gamma_{p}(\alpha) \Gamma_{p}(\beta)}\left\{{ }_{1} F_{1}(\alpha ; \alpha+\beta ;-\Lambda)\right\}^{-1} \operatorname{det}(Z)^{a-(p+1) / 2} \operatorname{etr}(-\Lambda U) \\
& \quad \times \operatorname{det}(U)^{-(a+b-\alpha)} \operatorname{det}(U-Z)^{b-(p+1) / 2} \operatorname{det}\left(I_{p}-U\right)^{\beta-(p+1) / 2}, \quad 0<Z<U<I_{p} .
\end{aligned}
$$

Now to obtain the marginal density of $Z$, we need to integrate $U$ in (4.10). Collecting terms containing $U$ and using the substitution $W=\left(I_{p}-Z\right)^{-1 / 2}\left(I_{p}-U\right)\left(I_{p}-Z\right)^{-1 / 2}$ with the Jacobian $J(U \rightarrow W)=\operatorname{det}\left(I_{p}-Z\right)^{(p+1) / 2}$, we get

$$
\begin{gathered}
\int_{0<Z<U<I_{p}} \operatorname{etr}(-\Lambda U) \operatorname{det}(U)^{-(a+b-\alpha)} \operatorname{det}(U-Z)^{b-(p+1) / 2} \operatorname{det}\left(I_{p}-U\right)^{\beta-(p+1) / 2} d U \\
=\operatorname{etr}(-\Lambda) \operatorname{det}\left(I_{p}-Z\right)^{b+\beta-(p+1) / 2} \int_{0<W<I_{p}} \operatorname{etr}\left\{\left(I_{p}-Z\right)^{1 / 2} \Lambda\left(I_{p}-Z\right)^{1 / 2} W\right\} \\
\quad \times \operatorname{det}(W)^{\beta-(p+1) / 2} \operatorname{det}\left(I_{p}-W\right)^{b-(p+1) / 2} \operatorname{det}\left(I_{p}-\left(I_{p}-Z\right) W\right)^{-(a+b-\alpha)} d W .
\end{gathered}
$$

Expanding etr $\left.\left(I_{p}-Z\right)^{1 / 2} \Lambda\left(I_{p}-Z\right)^{1 / 2} W\right\}$ and $\operatorname{det}\left(I_{p}-\left(I_{p}-Z\right) W\right)^{-(a+b-\alpha)}$ in series involving zonal polynomials and using (2.3), we obtain

$$
\begin{aligned}
& \operatorname{etr}\left\{\left(I_{p}-Z\right)^{1 / 2} \Lambda\left(I_{p}-Z\right)^{1 / 2} W\right\} \operatorname{det}\left(I_{p}-\left(I_{p}-Z\right) W\right)^{-(a+b-\alpha)} \\
& \quad=\sum_{k=0}^{\infty} \sum_{\alpha} \sum_{l=0}^{\infty} \sum_{\lambda} \frac{1}{k !} \frac{(a+b-\alpha)_{\lambda}}{l !} \sum_{\phi \in \kappa \cdot \lambda} \theta_{\phi}^{\kappa, \lambda} C_{\phi}^{\kappa, \lambda}\left(A W A^{\prime}, I_{p}-Z\right),
\end{aligned}
$$

where $A=\Lambda^{1 / 2}\left(I_{p}-Z\right)^{1 / 2}$. Substituting (4.12) in (4.11) and integrating $W$ using (2.6), we obtain

$$
\begin{aligned}
& \int_{0<Z<U<l_{p}} \operatorname{etr}(-\Lambda U) \operatorname{det}(U)^{-(a+b-\alpha)} \operatorname{det}(U-Z)^{b-(p+1) / 2} \operatorname{det}\left(I_{p}-U\right)^{\beta-(p+1) / 2} d U \\
&= \operatorname{etr}(-\Lambda) \operatorname{det}\left(I_{p}-Z\right)^{b+\beta-(p+1) / 2} \sum_{k=0}^{\infty} \sum_{\kappa} \sum_{l=0}^{\infty} \sum_{\lambda} \frac{1}{k !} \frac{(a+b-\alpha)_{\lambda}}{l !} \\
& \times \sum_{\phi \in \kappa \cdot \lambda} \theta_{\phi}^{\kappa, \lambda} \int_{0<W<I_{p}} \operatorname{det}(W)^{\beta-(p+1) / 2} \operatorname{det}\left(I_{p}-W\right)^{b-(p+1) / 2} \\
& \times C_{\phi}^{\kappa, \lambda}\left(A W A^{\prime}, I_{p}-Z\right) d W \\
&= \operatorname{etr}(-\Lambda) \operatorname{det}\left(I_{p}-Z\right)^{b+\beta-(p+1) / 2} \sum_{k=0}^{\infty} \sum_{\kappa} \sum_{l=0}^{\infty} \sum_{\lambda} \frac{1}{k !} \frac{(a+b-\alpha)_{\lambda}}{l !} \\
& \times \sum_{\phi \in \kappa \cdot \lambda} \theta_{\phi}^{\kappa, \lambda} \frac{\Gamma_{p}(\beta, \kappa) \Gamma(b)}{\Gamma_{p}(b+\beta, \kappa)} C_{\phi}^{\kappa, \lambda}\left(A A^{\prime}, I_{p}-Z\right)
\end{aligned}
$$

Integrating $U$ in (4.10) using (4.13), and simplifying the resulting expression using Kummer's relation we get the desired result. 
In above theorem if we take $\alpha=a+b$, then

$$
\begin{gathered}
\sum_{k=0}^{\infty} \sum_{\kappa} \sum_{l=0}^{\infty} \sum_{\lambda} \frac{1}{k !} \frac{(a+b-\alpha)_{\lambda}}{l !} \sum_{\phi \in \kappa \cdot \lambda} \theta_{\phi}^{\kappa, \lambda} \frac{(\beta)_{\kappa}}{(b+\beta)_{\kappa}} C_{\phi}^{\kappa, \lambda}\left(\Lambda\left(I_{p}-Z\right), I_{p}-Z\right) \\
=\sum_{k=0}^{\infty} \sum_{\kappa} \frac{(\beta)_{\kappa}}{(b+\beta)_{\kappa} k !} C_{\kappa}\left(\left(I_{p}-Z\right) \Lambda\right)={ }_{1} F_{1}\left(\beta ; b+\beta ; \Lambda\left(I_{p}-Z\right)\right)
\end{gathered}
$$

and the density of $Z$ reduces to

$$
\begin{aligned}
& \frac{\Gamma_{p}(a+b+\beta)}{\Gamma_{p}(a) \Gamma_{p}(b+\beta)}\left\{_{1} F_{1}(\beta ; a+b+\beta ; \Lambda)\right\}^{-1} \operatorname{det}(Z)^{a-(p+1) / 2} \\
& \quad \times \operatorname{det}\left(I_{p}-Z\right)^{b+\beta-(p+1) / 2}{ }_{1} F_{1}\left(\beta ; b+\beta ;\left(I_{p}-Z\right) \Lambda\right), \quad 0<Z<I_{p} .
\end{aligned}
$$

Furthermore, for $\Lambda=0, Z \sim B_{p}^{I}(a, b+\beta)$, a result derived by Rao [12] in the univariate case and Javier and Gupta [10] (also see Gupta and Nagar [8, 9]) in the matrix variate case.

THEOREM 4.7. Let $V \sim B_{p}^{I I}(a, b)$ and $U \sim K B_{p}(\alpha, \beta, \Lambda)$ be independent. Then the density of $Z=U^{1 / 2} V U^{1 / 2}$ is given by

$$
\begin{aligned}
& \frac{K(\alpha, \beta, \Lambda) \Gamma_{p}(\alpha+b) \Gamma_{p}(\beta)}{\beta(a, b) \Gamma_{p}(\alpha+\beta+b)} \operatorname{etr}(-\Lambda) \operatorname{det}(Z)^{a-(p+1) / 2} \operatorname{det}\left(I_{p}+Z\right)^{-(a+b)} \\
& \quad \times \sum_{k=0}^{\infty} \sum_{l=0}^{\infty} \sum_{\kappa} \sum_{\lambda} \frac{(a+b)_{\lambda}}{k ! l !} \sum_{\phi \in \kappa \cdot \lambda} \theta_{\phi}^{\kappa, \lambda} \frac{(\beta)_{\phi}}{(\alpha+\beta+b)_{\phi}} C_{\phi}^{\kappa, \lambda}\left(\Lambda,\left(I_{p}+Z\right)^{-1}\right), \quad Z>0 .
\end{aligned}
$$

PROOF. The joint density of $U$ and $V$ is given by

$$
\begin{aligned}
& \frac{K(\alpha, \beta, \Lambda)}{\beta(a, b)} \operatorname{etr}(-\Lambda U) \operatorname{det}(U)^{\alpha-(p+1) / 2} \operatorname{det}\left(I_{p}-U\right)^{\beta-(p+1) / 2} \\
& \quad \times \operatorname{det}(V)^{a-(p+1) / 2} \operatorname{det}\left(I_{p}+V\right)^{-(a+b)}, \quad 0<U<I_{p}, V>0 .
\end{aligned}
$$

Transforming $Z=U^{1 / 2} V U^{1 / 2}$ with $J(V \rightarrow Z)=\operatorname{det}(U)^{-(p+1) / 2}$ in above, we get the joint density of $U$ and $Z$ as

$$
\begin{aligned}
& \frac{K(\alpha, \beta, \Lambda)}{\beta(a, b)} \operatorname{etr}(-\Lambda U) \operatorname{det}(U)^{\alpha+b-(p+1) / 2} \operatorname{det}\left(I_{p}-U\right)^{\beta-(p+1) / 2} \\
& \quad \times \operatorname{det}(Z)^{a-(p+1) / 2} \operatorname{det}(U+Z)^{-(a+b)}, \quad 0<U<I_{p}, Z>0 .
\end{aligned}
$$

Now, writing $\operatorname{det}(U+Z)=\operatorname{det}\left(I_{p}+Z\right) \operatorname{det}\left(I_{p}-\left(I_{p}+Z\right)^{-1}\left(I_{p}-U\right)\right)$ and integrating out $U$, the density of $Z$ is obtained as

$$
\frac{K(\alpha, \beta, \Lambda)}{\beta(a, b)} \operatorname{det}(Z)^{a-(p+1) / 2} \operatorname{det}\left(I_{p}+Z\right)^{-(a+b)} \int_{0<U<l_{p}} \operatorname{etr}(-\Lambda U) \operatorname{det}(U)^{\alpha+b-(p+1) / 2}
$$




$$
\begin{aligned}
& \times \operatorname{det}\left(I_{p}-U\right)^{\beta-(p+1) / 2} \operatorname{det}\left(I_{p}-\left(I_{p}+Z\right)^{-1}\left(I_{p}-U\right)\right)^{-(a+b)} d U \\
= & \frac{K(\alpha, \beta, \Lambda)}{\beta(a, b)} \operatorname{etr}(-\Lambda) \operatorname{det}(Z)^{a-(p+1) / 2} \operatorname{det}\left(I_{p}+Z\right)^{-(a+b)} \int_{0<Y<I_{p}} \operatorname{etr}(\Lambda Y) \\
& \times \operatorname{det}\left(I_{p}-Y\right)^{\alpha+b-(p+1) / 2} \operatorname{det}(Y)^{\beta-(p+1) / 2} \operatorname{det}\left(I_{p}-\left(I_{p}+Z\right)^{-1} Y\right)^{-(a+b)} d Y \\
= & \frac{K(\alpha, \beta, \Lambda)}{\beta(a, b)} \operatorname{etr}(-\Lambda) \operatorname{det}(Z)^{a-(p+1) / 2} \operatorname{det}\left(I_{p}+Z\right)^{-(a+b)} \\
& \times \sum_{k=0}^{\infty} \sum_{l=0}^{\infty} \sum_{\kappa} \sum_{\lambda} \frac{1}{k !} \frac{(a+b)_{\lambda}}{l !} \sum_{\phi \in \kappa \cdot \lambda} \theta_{\phi}^{\kappa, \lambda} \int_{0<Y<I_{p}} \operatorname{det}(Y)^{\beta-(p+1) / 2} \\
& \times \operatorname{det}\left(I_{p}-Y\right)^{\alpha+b-(p+1) / 2} C_{\phi}^{\kappa, \lambda}\left(\Lambda Y,\left(I_{p}+Z\right)^{-1} Y\right) d Y, \quad Z>0,
\end{aligned}
$$

where the last step has been obtained by expanding $\operatorname{etr}(\Lambda Y)$ and $\operatorname{det}\left(I_{p}-\left(I_{p}+\right.\right.$ $\left.Z)^{-1} Y\right)^{-(a+b)}$ in series involving zonal polynomials and subsequently using (2.3). Finally, integration of $Y$ above using (2.5) yields the density of $Y$.

THEOREM 4.8. Let $S(p \times p)$ and $U(p \times p)$ be independent, $S \sim G_{p}\left(m, I_{p}\right)$ and $U \sim K B_{p}\left(\alpha, \beta, I_{p}\right)$. Define $Z=U^{-1 / 2} S U^{-1 / 2}$.Then the density of $Z$ is given by

$$
\begin{aligned}
& \frac{K\left(\alpha, \beta, I_{p}\right) \Gamma_{p}(\alpha+m) \Gamma_{p}(\beta)}{\Gamma_{p}(m) \Gamma_{p}(\alpha+\beta+m)} \operatorname{det}(Z)^{m-(p+1) / 2} \\
& \quad \times{ }_{1} F_{1}\left(\alpha+m ; \alpha+\beta+m ;-\left(I_{p}+Z\right)\right), \quad Z>0 .
\end{aligned}
$$

PROOF. Use of the transformation $Z=U^{-1 / 2} S U^{-1 / 2}$ with the Jacobian $J(S \rightarrow$ $Z)=\operatorname{det}(U)^{(p+1) / 2}$ yields the joint density of $Z$ and $U$ as

$$
\frac{K\left(\alpha, \beta, I_{p}\right)}{\Gamma_{p}(m)} \operatorname{etr}\left\{-\left(I_{p}+Z\right) U\right\} \operatorname{det}(Z)^{m-(p+1) / 2} \operatorname{det}(U)^{\alpha+m-(p+1) / 2} \operatorname{det}\left(I_{p}-U\right)^{\beta-(p+1) / 2},
$$

where $0<U<I_{p}, Z>0$. The result follows from the definition of the confluent hypergeometric function.

\section{Acknowledgment}

This research was supported by the Comité para el Desarrollo de la Investigación, Universidad de Antioquia research grant no. IN91CE.

\section{References}

[1] C. Armero and M. J. Bayarri, 'A Bayesian analysis of a queuing system with unlimited service', Technical Report No. 93-50, (Department of Statistics, Purdue University, 1993). 
[2] —-, 'A Bayesian analysis of a queuing system with unlimited service', J. Statist. Plann. Inference 58 (1997), 241-264.

[3] Y. Chikuse, 'Distributions of some matrix variates and latent roots in multivariate Behrens-Fisher discriminant analysis', Ann. Statist. (2) 9 (1981), 401-407.

[4] A. G. Constantine, 'Some non-central distribution problems in multivariate analysis', Ann. Math. Statist. 34 (1963), 1270-1285.

[5] A. W. Davis, 'Invariant polynomials with two matrix arguments extending the zonal polynomials: applications to multivariate distribution theory', Ann. Inst. Statist. Math. 31 (1979), 465-485.

[6] - - 'Invariant polynomials with two matrix arguments extending the zonal polynomials', in: Multivariate analysis V (ed. P. R. Krishnaiah) (North-Holland, Amsterdam, 1980) pp. 287-299.

[7] M. B. Gordy, 'Computationally convenient distributional assumptions for common-value actions', Comput. Econ. 12 (1998), 61-78.

[8] A. K. Gupta and D. K. Nagar, 'Matrix variate beta distribution', Int. J. Math. Math. Sci. (7) 24 (2000), 449-459.

[9] - Matrix variate distributions (Chapman and Hall/CRC, Boca Raton, 2000).

[10] W. R. Javier and A. K. Gupta, 'On generalized matrix variate beta distributions', Statistics (4) 16 (1985), 549-558.

[11] K. W. Ng and S. Kotz, 'Kummer-Gamma and Kummer-Beta univariate and multivariate distributions', Research Report No. 84, (Department of Statistics, The University of Hong Kong, Hong Kong, 1995).

[12] C. R. Rao, Advanced statistical methods in biometric research (John Wiley and Sons, New York, 1952).

Departamento de Matemáticas

Universidad de Antioquia

Medellín, A. A. 1226

Colombia

e-mail: nagar@matematicas.udea.edu.co
Department of Mathematics and Statistics

Bowling Green State University Bowling Green, Ohio 43403-0221

USA

e-mail: gupta@bgnet.bgsu.edu 
